EPiC Series in Engineering
Volume 1, 2018, Pages 36-40
ReVo 2017: Laval Virtual ReVolution 2017
"Transhumanism++"

\title{
"HADO" as Techno Sports was born by the fusion of IT technology and sports
}

\author{
Hitoshi Araki ${ }^{1}$, Hiroshi Fukuda ${ }^{1}$, Takuma Motoki ${ }^{1}$, \\ Tsuyoshi Takeuchi ${ }^{1}$, Naoki Ohta ${ }^{1}$, Ryosuke Adachi ${ }^{1}$, Hiroshi Masuda ${ }^{1}$, \\ Yasuyuki Kado ${ }^{1}$, Yoshiki Mita ${ }^{1}$, Daisuke mizukami ${ }^{1}$, Nayu Kakeya ${ }^{1}$ \\ ${ }^{1}$ meleap inc. \\ hitoshi.araki@meleap.com
}

\begin{abstract}
"HADO" is the combination of Augmented Reality (AR) technology, motion sensor, Head-mounted display (HMD), smartphone, and sports that creates a whole new experience that we call "Techno Sports". By wearing the arm sensor in the arm and HMD to the head, adding the AR technology, HADO actualize magical worlds everyone dreams of as a child. Shoot energy balls to smash opponents and use the shield to prevent opponent's attacks!! Think strategic, move like a lightning, and coordinate team play to flight your opponents. This is a HADO Players vs. Players battle!!
\end{abstract}

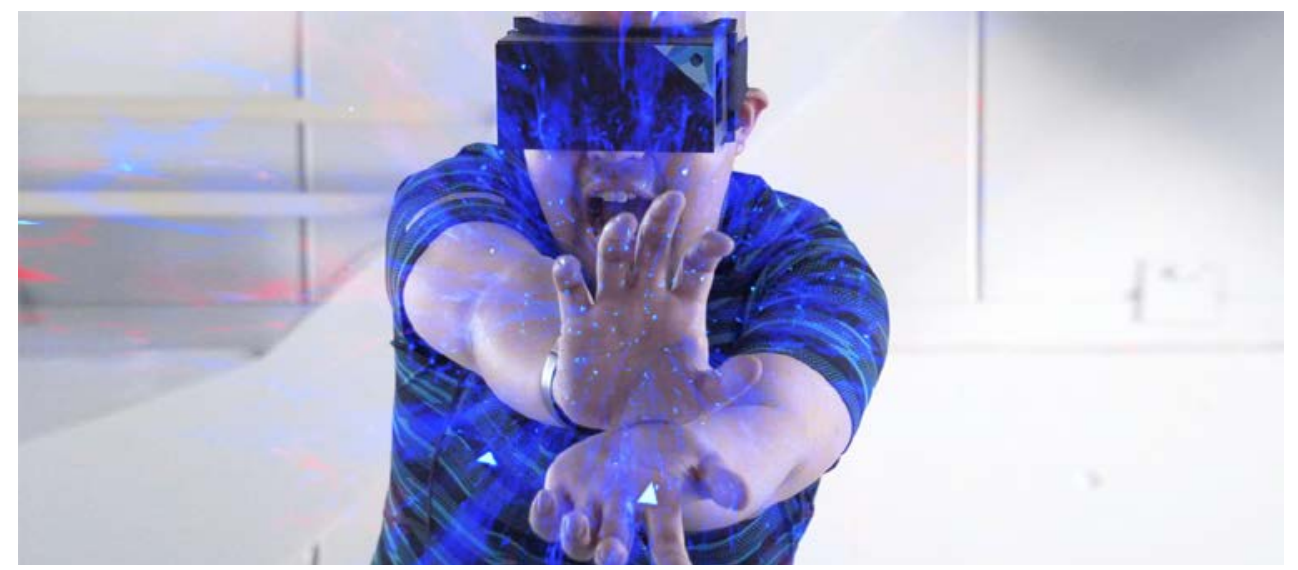

Figure 1. Everyone can shoot the magical skills by the "HADO" in the real world. 


\section{Introduction}

\subsection{About Techno Sports}

The birth of motor sports is caused by a rise of the automobile industry. At last, some evolutions of wearable devices are possible to follow human movements. These new technologies have the possibility to create new sports. Techno Sports that we call are born by fusion of Information Technology and Sports. Players move their bodies and enjoy sports supported by wearable devices and communication technology.

In a similar approach, there are Superhuman Sports [1]. Superhuman Sports are new kind of sports with advanced technologies. The Superman Sports Association is engaged in sports, technology and culture, and is developing sports for the purpose of creating a "human-machine unity" sports which is carried out by a person and a machine together. HADO has been adopted as an official Superman Sport.

\subsection{About HADO}

When I was a child, I dreamed of shooting some magical skills. Now, the dream comes true by HADO. HADO is a techno sports developed by meleap inc. . With the HMD inserted a smartphone and the wristband device, the players can multi-play with moving around.

There are three different types of content in HADO. "Players vs. Players" is fighting against two teams of up to three players, "vs. Monsters" is fighting to monsters in cooperation with up to 8 players, and "HADO Kart" is enjoying kart ride with HADO.

The player shoots the attacking skill called Energy ball and creates the shield skill called barrier by performing a specific arm motion. Players can enjoy the experiences by the real time rendering of these skill-effects as CG on the camera image of the smartphone. In addition, the players fight using the team play strategies such as the share of barrier activated by other team member.

"VOID"[2] and "ZERO LATENCY"[3] can be cited as a similar service. It's different in that they using VR technology and heavy equipment like gun. The experiences are like FPS game. HADO need so light equipment than theirs because of no backpack PC. HADO players can move sharply just like other sports.

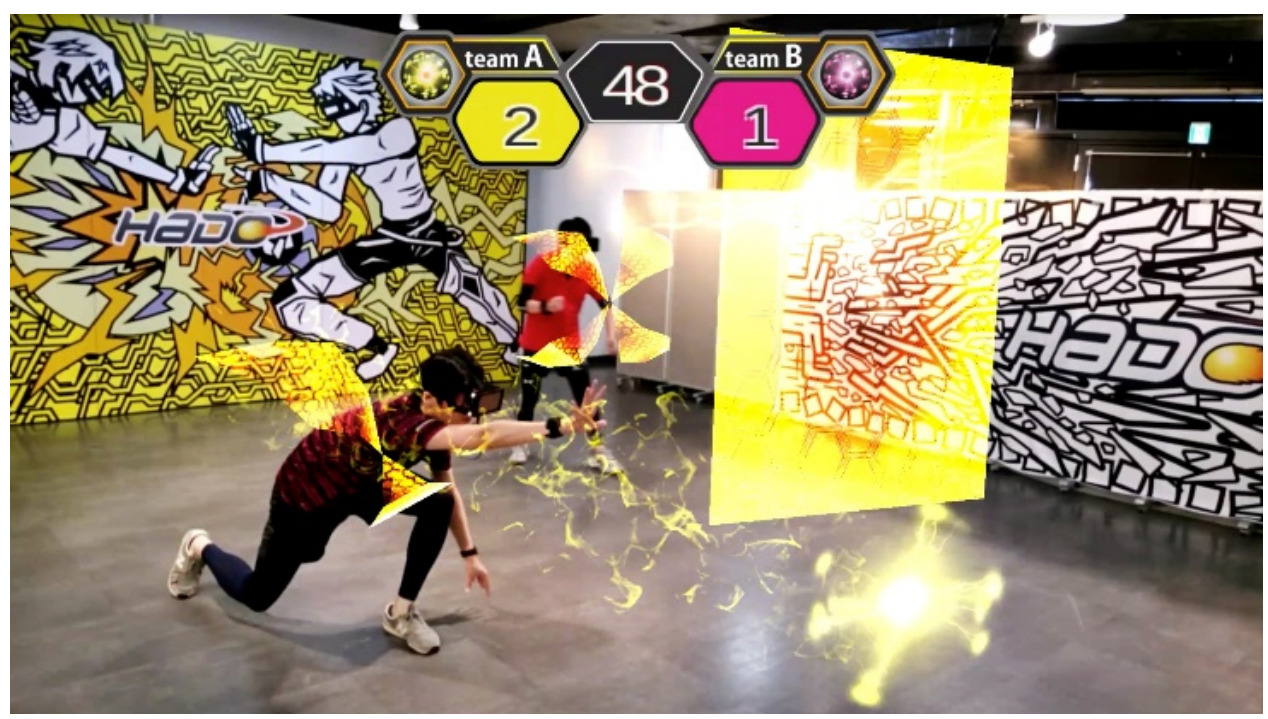

Figure 1. The play capture of HADO 


\section{HADO Components}

\subsection{The equipment of HADO}

HADO players wear the HMD inserted a smartphone and the wristband device as a motion sensor.

The HMD is made by ourselves for AR experience. For the purpose of player's mobility, the HMD is made as light as possible. Besides, setting up the sidelight and adopting transparent material to bottom spread the angle of view spuriously. From these points, player can look around and move more easily than using the HMD for VR. As a side effect, VR sickness is few due to reduced immersion.

Player's arm motion is recognized by the motion sensor to activate the skills. Especially, Using a cut Myo[4] equipped with the three-axis acceleration sensor and three-axis gyro sensor, player can shoot the skill irrespective of age and arms thickness.

Just using light and mobile HMD and motion sensor, now anyone can activate the skills.

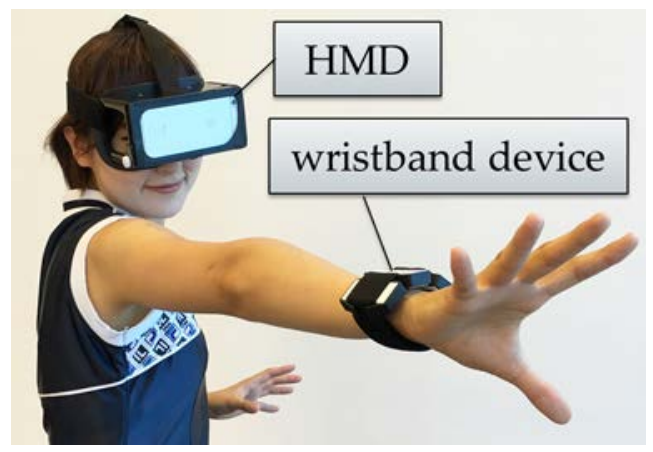

Figure 2. The equipment of HADO

\subsection{Play Field}

Official HADO Play Field is $10 \mathrm{~m}$ wide by $7 \mathrm{~m}$ deep. According to the situation, the play field size is adjusted. The AR Backdrop is set up at the both wide edge of the field. And the third point view smartphone installed HADO app is located at the out of the field. The smartphone is connected to TV monitor or projector for displaying AR world to the audience. The audience can cheer players by the grasp of game situation in real time. Beside of the play field, there is the local network server and the wireless LAN base station. Each player's position data acquired by AR Technology are shared and updated in real time through the wireless network. From these points, the player can look and avoid the other player's skill.

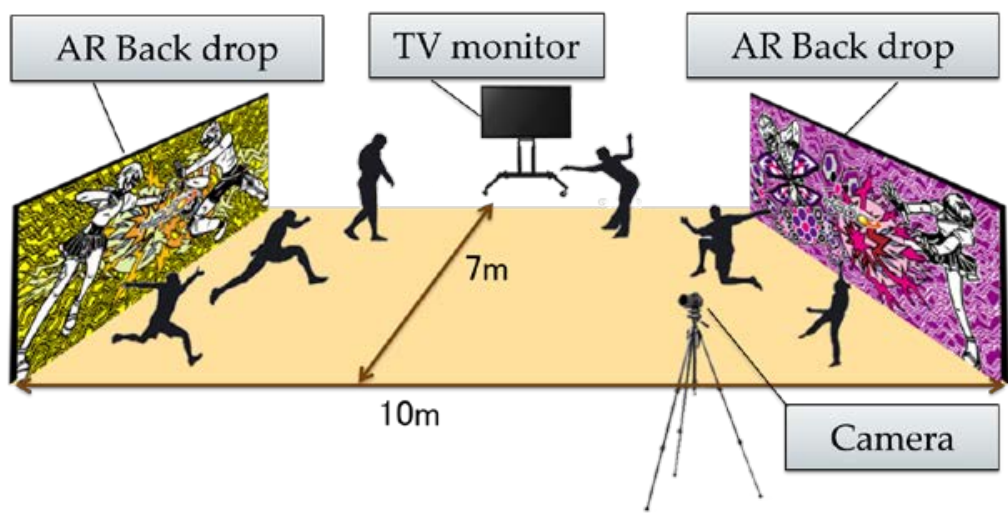

Figure 3. The play field of HADO 


\section{Results and Discussion}

To As HADO is in commercial use from Dec 2015. HADO player's fatigue is less by the lighter equips and the short playing time (80 180 second/play). All genders and generations can play (6 years old and above). Besides the development of Monocular HMD, pre-school children can play HADO.

HADO is running as attraction or activity at mainly leisure facilities, shopping mall. For instance, domestic permanent use case is HUIS TEN BOSCH[5], NAMJA TOWN[6] and VR Center at AEON LakeTown[7]. Overseas permanent use case is JOYPOLIS at Qingdao China[8]. Temporary event use case is more than 70 locations. Depends on the situation, max capacity per day is 1,000 players. Over 600,000 people play HADO so far. Various players (friends, families especially children, couples, cosplayer) enjoy HADO for each purpose (Leisure, Sports, Exercise, Cosplay), core players play so many times.

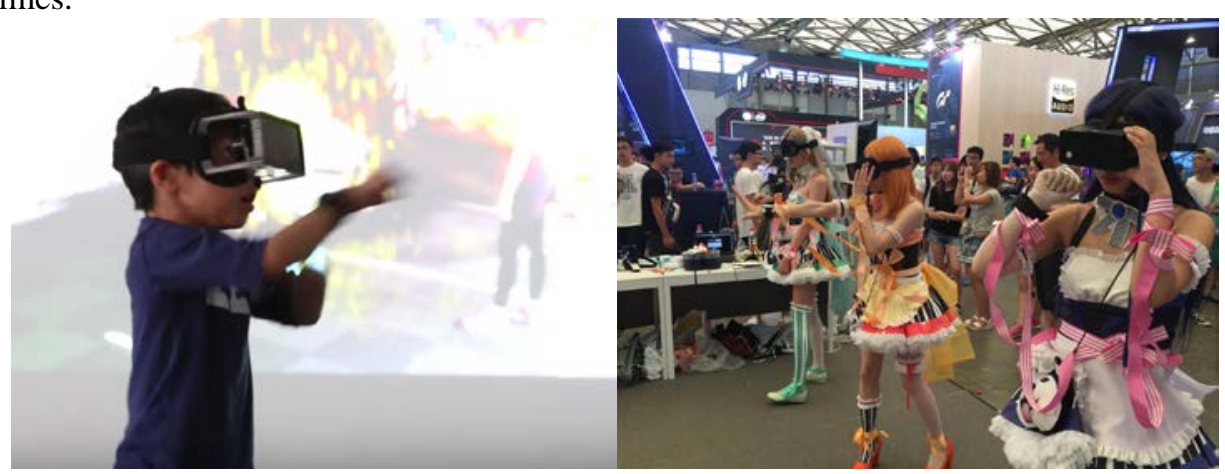

Figure 4. The various players of HADO

\section{Conclusions}

For In summary, HADO actualize magical worlds everyone dreams of as a child by wearing the arm sensor in the arm and HMD to the head, adding the AR technology. HADO is spreading not only Japan but overseas as Techno Sports.

In 26 Nov 2016, HADO WORLD CUP[9] was held at Tokyo as the world's first techno sports competition with prize money. The applying team number is almost 4 times to limit on participation, 16 teams and about 50 players participated and fought a hot fight. Approximately 33,000 audiences cheered mainly on web live streaming. HADO WORLD CUP will be held every year, and next time we'd like to invite players from Asia, North America, and Europe.

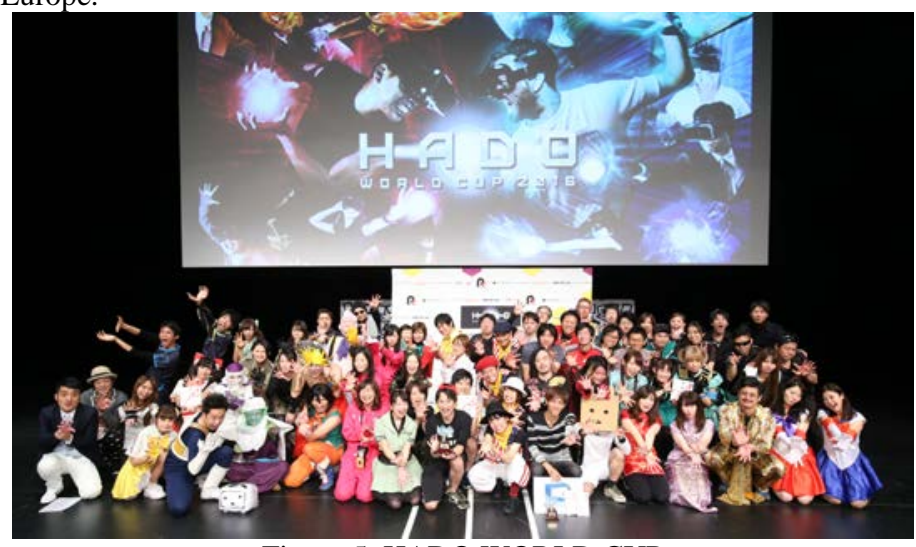

Figure 5. HADO WORLD CUP 


\section{References}

[1] Superhuman Sports: http://superhuman-sports.org/

[2] VOID: https://thevoid.com/

[3] ZERO LATENCY: https://www.zerolatencyvr.com/

[4] Myo: https://www.myo.com/

[5] HUIS TEN BOSCH: https://www.huistenbosch.co.jp/

[6] NAMJA TOWN: http://event.namco.co.jp/namja/en/

[7] VR Center at AEON LakeTown: https://www.vrcenter.jp/

[8] Qingdao JOYPOLIS: http://www.segajoypolis.cn/

[9] HADO WORLD CUP: http://meleap.com/worldcup/ 